\title{
Facilitation of Extinction Learning for Contextual Fear Memory by PEPA: A Potentiator of AMPA Receptors
}

\author{
Ko Zushida, ${ }^{1}$ Mikako Sakurai, ${ }^{1}$ Keiji Wada, ${ }^{1,2}$ and Masayuki Sekiguchi ${ }^{1,2}$ \\ ${ }^{1}$ Department of Degenerative Neurological Diseases, National Institute of Neuroscience, National Center of Neurology and Psychiatry, Kodaira, Tokyo 187- \\ 8502, Japan, and ${ }^{2}$ Core Research for Evolutional Science and Technology, Japan Science and Technology Agency, Kawaguchi, Saitama 322-0012, Japan
}

Contextual fear memory is attenuated by the re-exposure of mice to the context without aversive stimulus. This phenomenon is called extinction. Here, we report that a potentiator of AMPA receptors, 4-[2-(phenylsulfonylamino)ethylthio]-2,6-difluorophenoxyacetamide (PEPA), potently facilitates extinction learning in mice. C57BL/6J mice were exposed to novel context and stimulated by electrical footshock. After $24 \mathrm{~h}$ (extinction training) and $72 \mathrm{~h}$ (extinction test), the mice were repeatedly exposed to the context without footshock and the duration of their freezing response was measured. The duration of freezing response in the extinction test was consistently shorter than the value in extinction training. Intraperitoneal injection of PEPA 15 min before extinction training remarkably reduced the duration of freezing responses during the extinction training and test, compared with the vehicle-injected control mice. This action of PEPA on extinction was dose-dependent and inhibited by NBQX (1,2,3,4-tetrahydro-6-nitro-2,3-dioxo-benzo[f]quinoxaline-7sulfonamide), an AMPA receptor antagonist. PEPA had no effect on acquisition and consolidation of fear memory itself. Electrophysiological studies suggested that PEPA activates the neural network much more potently in the medial prefrontal cortex ( $\mathrm{mPFC}$ ) than in the basolateral amygdala and hippocampal CA1 field. Quantitative PCR studies suggested the pronounced expression of PEPA-preferring AMPA receptor subunits (GluR3 and GluR4) and a splice variant (flop) in the MPFC. An intra-mPFC injection of PEPA facilitated the extinction much more potently than an intra-amygdala injection of PEPA did. These results suggest that PEPA facilitates extinction learning through AMPA receptor activation mainly in the $\mathrm{MPFC}$.

Key words: AMPA receptor; potentiator; PEPA; extinction; fear; medial prefrontal cortex

\section{Introduction}

Contextual conditioned fear is acquired in response to an aversive stimulus such as electrical shock [unconditioned stimulus (US)] that is loaded during exposure of mice to a conditioned stimulus (CS), such as novel context. Re-exposure to the CS triggers a conditioned response (CR) such as defensive freezing behavior (for review, see Fanselow 1998; LeDoux 2000; Davis and Whalen, 2001; Maren 2001). However, if the CS is presented without the US, the CR gradually declines. This phenomenon is called "extinction" of conditioned fear memory (for review, see Myers and Davis, 2002; Maren and Quirk 2004). It is thought that extinction is a result of new learning that forms "inhibitory association" between the CS and the US, which suppresses the emergence of the CR (Bouton et al., 1993; Berman and Dudai, 2001; Bouton, 2002; for review, see Myers and Davis, 2002). Connections between the medial prefrontal cortex (mPFC) and the amygdala are thought to be neural bases for extinction (Morgan

\footnotetext{
Received Sept. 5, 2006; revised Nov. 17, 2006; accepted Nov. 21, 2006.

This work was supported in part by Grants-in-Aid for Scientific Research from the Ministry of Health, Labour and Welfare of Japan, Grants-in-Aid for Scientific Research from the Ministry of Education, Culture, Sports, Science and Technology of Japan, the Program for Promotion of Fundamental Studies in Health Sciences of the National Institute of Biomedical Innovation, and a grant from the Japan Science and Technology Agency.

Correspondence should be addressed to Masayuki Sekiguchi, Department of Degenerative Neurological Diseases, National Institute of Neuroscience, National Center of Neurology and Psychiatry, 4-1-1 Ogawahigashi, Kodaira, Tokyo 187-8502, Japan. E-mail: elec1@ncnp.go.jp or sekiguch@ncnp.go.jp.

D0I:10.1523/JNEUROSCI.3842-06.2007

Copyright $\odot 2007$ Society for Neuroscience $\quad$ 0270-6474/07/270158-09\$15.00/0
}

et al., 1993; Morgan and LeDoux, 1995; Garcia et al., 1999, Quirk et al., 2000; Rosenkranz and Grace, 2001; Quirk et al., 2003).

Studies using positron emission tomography show dysfunction of the medial and orbital PFC during provocation of posttraumatic stress disorder (PTSD) symptoms (Bremner, 1999). Given that the mPFC plays a crucial role in extinction, a drug that could activate the $\mathrm{MPFC}$ has the potential to facilitate exposurebased cognitive behavioral therapy (CBT) for psychiatric disorders, such as PTSD, because CBT depends on the process of fear extinction (for review, see Davis et al., 2006). D-Cycloserine, an agonist and potentiator of the NMDA subtype of glutamate receptors, facilitates extinction learning for fear memory (Walker et al., 2002). This compound is thought to facilitate inhibitory association between the CS and US within the amygdala (Walker et al., 2002; for review, see Davis et al., 2006). The AMPA receptor is another major subtype of glutamate receptors and mediates fast excitatory synaptic transmission in the majority of the central glutamatergic synapses (Hestrin et al., 1990; Ozawa et al., 1991). Each AMPA receptor subunit exists in flip and flop variants generated by alternative splicing, and their expression is regulated both regionally and developmentally (Sommer et al., 1990). The activation of AMPA receptors releases NMDA receptors from voltage-dependent magnesium block (Mayer et al., 1984); the role of AMPA receptors in extinction learning, however, is obscure.

In the present study, we investigated the effects of 4-[2-(phenylsulfonylamino) ethylthio]-2,6-difluorophenoxyacetamide 
(PEPA), a flop splice variant and GluR3/4-prefrerring potentiator of AMPA receptors (Sekiguchi et al., 1997, 1998), on extinction learning. Potentiators for AMPA receptors are small chemical compounds that enhance AMPA receptor channel activity by modulating receptor kinetics such as desensitization and deactivation [in the case of PEPA (Sekiguchi et al., 2002)]. Some of these compounds reportedly enhance synaptic response by acting on AMPA receptors [in the case of PEPA (Nakagawa et al., 1999)] (for review, see O'Neill et al., 2004). Here, we found that PEPA potently facilitates extinction learning through activation of AMPA receptors mainly in the $\mathrm{mPFC}$.

\section{Materials and Methods}

Animals. Male C57BL/6J mice were purchased from Clea (Tokyo, Japan) and used in experiments after habituation to the environment of our Institute's animal center for 1 week. The mice were housed five per cage under controlled conditions of temperature $\left(25 \pm 1^{\circ} \mathrm{C}\right)$ and lighting $(12$ $\mathrm{h}$ light/dark cycle) and provided with food and water ad libitum. The mice were 8-12 weeks old at the beginning of experiments. The experiments were performed in strict accordance with the regulations of the National Institute of Neuroscience (Japan) for animal experiments.

Behavioral procedures. Contextual fear conditioning was performed using the method reported previously (Takeda et al., 2003) with slight modifications. Briefly, a mouse was introduced to the operation box $(20 \times 20 \mathrm{~cm}$ bottom, $35 \mathrm{~cm}$ high, inside a soundproof box $)$ with a stainless grid floor. After $10 \mathrm{~s}$, electrical footshock was delivered from the grid for $360 \mathrm{~s}$ ( $1.3 \mathrm{~mA} ; 1 \mathrm{~s}$ duration; $10 \mathrm{~s}$ interval; scrambled). The similar stimulus condition, in particular, a condition where multiple electrical shocks are given, is also used in the study to examine the involvement of the mPFC in behavioral control against stress (Amat et al., 2005). Ten seconds after the end of electrical shock, the mouse was removed from the box and returned to his home cage. The behavior of the mouse was recorded using a digital video camera on the ceiling of the soundproof box. The illumination on the floor of the box was 230 lux. Complete immobilization of the mouse, except for respiration, was regarded as the freezing response (Blanchard and Blanchard, 1972). The freezing response was scored as the total time the mouse spent in freezing during the $360 \mathrm{~s}$ test session. The total time the mouse spent in freezing during each $60 \mathrm{~s}$ of the $360 \mathrm{~s}$ test session was plotted for the time-course analysis. The timing of exposure of mice to the operation box varied between experiments and is described in Results. PEPA (Sigma, St. Louis, MO, or kindly supplied from Nihonsuian, Japan) was dissolved into the vehicle consisting of $0.45 \% \mathrm{NaCl}$ and $33 \%$ (2-hydroxypropyl)- $\beta$-cyclodextrin, and injected intraperitoneally $(0.01 \mathrm{ml} / \mathrm{g}$ body weight $)$ into the mice unless otherwise noted. 1,2,3,4-tetrahydro-6-nitro-2,3-dioxo-benzo[f]quinoxaline-7-sulfonamide (NBQX) disodium salt (Sigma) was dissolved in saline $(0.9 \% \mathrm{NaCl})$. The timing of the injection varied between experiments and is described in Results.

Open-field tests were performed in an arena $($ a $50 \times 50 \mathrm{~cm}$ white field surrounded by a 40 -cm-high white wall, illuminated with 80 lux) that was placed in the same soundproof box described above. Mice were placed at the periphery of the arena, and for $5 \mathrm{~min}$ the behavior of mice was recorded into a computer using a digital video camera. Locomotion and the time the mice spent at a perimeter (i.e., within $10 \mathrm{~cm}$ of the wall) were calculated from this record by Image OF (O'Hara, Tokyo, Japan), a modified software based on the public domain of NIH Image.

The hole-board test was performed using the method reported previously (Takeda et al., 1998) with slight modifications. The hole-board apparatus $(50 \times 50 \mathrm{~cm}$ gray floor with four holes $3 \mathrm{~cm}$ in diameter, equally spaced, and a $40 \mathrm{~cm}$-high wall, illuminated with $170 \mathrm{lux}$ ) was placed in the soundproof box. Mice were placed in the center of the hole board, allowed to freely explore the apparatus for $5 \mathrm{~min}$, and headdipping behavior was counted by monitoring the mouse behavior using a digital video camera on the ceiling of the soundproof box.

Electrophysiology. Each male mouse (8-12 weeks old) was anesthetized with halothane and the brain was removed quickly. When recordings were performed from the $\mathrm{mPFC}$ or basolateral amygdala (BLA), coronal brain slices (300 $\mu \mathrm{m}$ thick) were prepared using a Vibratome 3000 (Vi- bratome, St. Louis, $\mathrm{MO}$ ) in artificial CSF [ACSF; containing the following (in mM): $125 \mathrm{NaCl}, 4.4 \mathrm{KCl}, 1.5 \mathrm{MgSO}_{4}, 1.0 \mathrm{NaH}_{2} \mathrm{PO}_{4}, 26 \mathrm{NaHCO}_{3}$, 10 glucose, $2.5 \mathrm{CaCl}_{2}$, pH 7.4, 290-300 mOsm/l]. When recordings were performed from the CA1 field, the hippocampus was isolated from the brain and transverse slices (400 $\mu \mathrm{m}$ thick) were prepared as described previously (Nakagawa et al., 1999; Takamatsu et al., 2005) using a Vibratome 3000 in a sucrose-based cutting solution [containing the following (in mM): 234 sucrose, $25 \mathrm{KCl}, 1.25 \mathrm{NaH}_{2} \mathrm{PO}_{4}, 10 \mathrm{MgSO}_{4}, 26$ $\mathrm{NaHCO}_{3}, 11$ glucose, $\left.0.5 \mathrm{CaCl}_{2}\right]$. The slices were maintained at room temperature in ACSF continuously bubbled with $95 \% \mathrm{O}_{2} / 5 \% \mathrm{CO}_{2}$. A slice was transferred to the recording chamber and was continuously perfused $(3 \mathrm{ml} / \mathrm{min})$ with ACSF maintained at $28-32^{\circ} \mathrm{C}$. Somatic wholecell patch-clamp recordings were made with an EPC-9 (HEKA, Lambrecht/Pfalz, Germany) amplifier for the neurons identified using infrared differential contrast videomicroscopy (Hamamatsu Photonics, Hamamatsu, Japan) with an upright microscope (Axioscop; Zeiss, Oberkochen, Germany). In the mPFC slices, the layer V pyramidalshaped neurons with thick primary apical dendrites toward the midline direction were selected for the recording. The location of the recorded neurons was verified using a brain atlas (Paxinos and Franklin, 2001) and confirmed to be in the regions of the cingulate and prelimbic cortices. The pyramidal-shaped neurons in the BLA and CA1 pyramidal layer were selected for recording. In all three regions, the neurons that were selected for recording showed the spike-frequency adaptation when suprathreshold depolarization pulses were applied. The patch electrodes (6-10 M $\Omega$ ) were filled with solution containing the following (in $\mathrm{mm}$ ): $132 \mathrm{~K}$ gluconate, $3 \mathrm{KCl}, 10$ HEPES, 0.5 EGTA, $1 \mathrm{MgCl}_{2}$, 12 sodium phosphocreatine, 3 ATP magnesium salt, $0.5 \mathrm{GTP}, \mathrm{pH} 7.4$, with $\mathrm{KOH}$, 285-290 mOsm/l. Because the reversal potential of $\mathrm{GABA}_{\mathrm{A}}$ receptormediated synaptic currents was estimated between -75 and $-85 \mathrm{mV}$ when this pipette solution was used, we used a holding potential of -80 $\mathrm{mV}$ in the whole-cell clamp mode to minimize the contribution of $\mathrm{GABA}_{\mathrm{A}}$ currents in the synaptic response. The signal was digitized at 1 point $/ 50 \mu$ s and stored using Pulse/Pulsefit (HEKA). The resting membrane potential of the cells used in the analysis was between -57 and -75 $\mathrm{mV}$ and the series resistance was 3-20 $\mathrm{M} \Omega$. Input resistance was continuously monitored during recordings (every $30 \mathrm{~s}$ ), and cells with a large drift in the value (more than $\pm 15 \%$ from the initial value) were removed from the analysis. Electrical stimulation was applied to elicit synaptic currents through a metal bipolar electrode positioned on layer II (mPFC), external capsule (BLA), or stratum radiatum on the border of the CA2 and CA3 fields (CA1). The stimulation was performed with a constant current mode (20-100 $\mu$ s duration). All drugs used in electrophysiological experiments were applied to slices by perfusion. PEPA was dissolved in dimethylsulfoxide (DMSO) at 2, 10, 50, and $150 \mathrm{~mm}$ and diluted with ACSF to prepare 2, 10, 50, and $150 \mu \mathrm{M}$ solutions on the day of use (the final DMSO concentration was $0.1 \%$ ). Cyclothiazide (Sigma) solution was prepared similarly. These drugs were applied for $10 \mathrm{~min}$ during continuous monitoring of synaptic currents (interstimulus inter$\mathrm{val}, 30 \mathrm{~s}$ ). The $5-10$ responses just before the application, during minutes 5-10 from the start of the application and after the application, were respectively averaged, and these traces were compared. To assess the effects of PEPA, we measured the area under the baseline (AUB; ms/pA) using AxoGraph version 3 (Molecular Devices, Union City, CA), and the effect was expressed as an "AUB ratio," which was obtained by dividing the AUB value during the application of PEPA by the value before the application of PEPA. Other chemicals, including CNQX, were purchased from Sigma.

Quantitative PCR. To isolate RNA from the $\mathrm{mPFC}$, we first prepared coronal brain slices (300 $\mu \mathrm{m}$ thickness) including the mPFC (near the bregma $+2.3 \mathrm{~mm}$ level). The region of the $\mathrm{mPFC}$ (mainly consisting of the prelimbic, medial orbital, and cingulate cortices) was manually isolated from slices under a stereoscopic microscope during which the slices were incubated in ACSF continuously bubbled with $95 \% \mathrm{O}_{2} / 5 \% \mathrm{CO}_{2}$. In the case of the amygdala, we also prepared similar brain slices including the BLA (near the bregma $-1.7 \mathrm{~mm}$ level). The delta region lying between the external capsule (mainly consisting of the BLA and LA) was cutoff from the slices and was saved for isolation of RNA. For the hippocampus, it was isolated from the brain, and transverse slices (400 $\mu \mathrm{m}$ 
thickness) were prepared. These slices used for the RNA isolation without additional microdissection. Two to three slices were combined per each region, and total RNAs were prepared from this combined tissue sample using a Mini RNA isolation kit (Zymo Research, Orange, CA). The RNA solutions were treated with RNase-free DNase I (Ambion, Austin, TX) to digest contaminated DNA. After inactivation of DNase I, single-strand cDNA synthesis was performed using the Superscript II cDNA synthesis kit (Invitrogen, Gaithersburg, MD) with oligo(dT) ${ }_{12-18}$ primers. This single-strand cDNA sample was used in quantitative PCR after digestion of RNA with RNase H. Quantitative PCR was performed with an Applied Biosystems (Foster City, CA) 7700 real-time PCR unit using the SYBR green premix and a primer set specific for each gene. $\beta$-actin sequence was used as an internal standard. The primer sets used to detect each gene are listed in supplemental Table 1 (available at www.jneurosci.org as supplemental material). The amplification efficiencies of GluR gene fragments by these eight primer sets were confirmed to be almost identical by checking the efficiency with a known concentration of template plasmid carrying each GluR gene fragment (including the amplification region). Also, the absence of any detectable cross-reactivity was confirmed by performing PCR, for example, using a flop-specific primer set and a plasmid carrying a fragment for a flip variant. The $\Delta \mathrm{Ct}$ value (Ct value of GluR-amplification minus the $\mathrm{Ct}$ value of $\beta$-actin amplification in the same sample) was calculated in each sample, and the relative expression of GluR to $\beta$-actin was expressed as a $2^{-\Delta \mathrm{Ct}}$ value. The percentage of a flop variant was calculated, for example in the case of GluR1, by dividing the $2^{-\Delta \mathrm{Ct}}$ value for GluR1-flop by the sum of those values for GluR1-flip and GluR1-flop.

Microinjection. Each male mouse (8-12 weeks old) was anesthetized with pentobarbital (50 mg/kg, i.p.) and affixed to the brain stereotaxic apparatus (Narishige Instruments, Tokyo, Japan). In the case of the mPFC injection, a single stainless-steel guide cannula (Eicom, Kyoto, Japan) was impaled into the brain so that its tip was positioned near the $\mathrm{mPFC}$ (anteroposterior, $2.10 \mathrm{~mm}$ from bregma; lateral, $0 \mathrm{~mm}$; ventral, $-1.71 \mathrm{~mm}$ ). In the case of the amygdala injection, the guide cannulas were bilaterally impaled (anteroposterior, $-1.22 \mathrm{~mm}$ from bregma; lateral $\pm 2.83 \mathrm{~mm}$; ventral, $-4.00 \mathrm{~mm}$ ). The cannulas were held in place with acrylic dental cement. A dummy cannula was inserted into the guide cannula to prevent clogging. Microinjection $(0.2 \mu \mathrm{l}$ per one individual $)$ of PEPA $(0.1 \mu \mathrm{g} / \mu \mathrm{l})$ or vehicle (ACSF) was performed on day 2 or 3 after surgery. For microinjection, the dummy cannula was removed from the guide cannula, and a 28 gauge injection cannula, extending $0.5 \mathrm{~mm}$ from the tip of the guide cannula, was inserted under anesthesia with diethyl ether. The injection cannula was connected via Teflon tubing to a micro syringe (Hamilton Company, Reno, NV) driven by a micro infusion pump (WPI, Sarasota, FL). The injection rate was $0.05 \mu \mathrm{l} / \mathrm{min}$. The injection cannula was left in position for an additional 2 min before withdrawal. Extinction training was performed $30 \mathrm{~min}$ after injection. After the behavioral test, mice were anesthetized with halothane and microinjected into the mPFC with $0.2 \mu \mathrm{l}$ of bromophenol blue. The brain was isolated and sectioned (400 and $120 \mu \mathrm{m}$ for the mPFC and amygdala, respectively) using a Vibratome 3000. Cannula location was assessed under a light microscope.

Statistical analysis. The statistical significance of differences among data groups was assessed using one-way measure ANOVA with Bonferroni/Dunn post hoc analysis or using the two-tailed Student's $t$ test.

\section{Results}

PEPA injected before extinction training facilitates extinction learning for fear memory

First, we investigated the effects of PEPA on the extinction of fear memory. For this purpose, mice were trained to form contextual fear memory by loading electrical footshock in an operation box. At $24 \mathrm{~h}$ postconditioning, mice were re-exposed to the operation box for $360 \mathrm{~s}$ without footshock. In the present study, we called this first re-exposure "extinction training" (Fig. 1A). Mice were injected with PEPA (3, 10, and $30 \mathrm{mg} / \mathrm{kg}$, i.p.) or vehicle $15 \mathrm{~min}$ before this extinction training (Fig. $1 A$ ). The vehicle-treated control mice displayed a severe freezing response during extinction training (Fig. $1 B$ ). Injection of PEPA reduced the duration of freezing in a dose-dependent manner (Fig. $1 B$ ), but statistical significance was obtained only in mice injected with $30 \mathrm{mg} / \mathrm{kg}$ PEPA $[161.3 \pm 16.8 \mathrm{~s}(n=11)$, against the control value of $238.5 \pm 15.8 \mathrm{~s}(n=9) ; p=0.002$; Bonferroni/Dunn test]. After this extinction training, mice were returned to their home cage; at $72 \mathrm{~h}$ after the initial conditioning, they were again exposed to the operation box (without footshock). We called this second reexposure the "extinction test" (Fig. 1A). The duration of the freezing response in this extinction test was significantly shorter in all four groups when compared with the respective values measured during extinction training (Fig. $1 C)(p<0.001$ in the control and in mice injected with 3 or $30 \mathrm{mg} / \mathrm{kg}$ PEPA, and $p=$ 0.007 in mice injected with $10 \mathrm{mg} / \mathrm{kg}$ PEPA; Bonferroni/Dunn test), suggesting that our experimental protocol could detect the extinction of fear memory. In mice injected with $30 \mathrm{mg} / \mathrm{kg}$ PEPA, the duration of the freezing response was $74.0 \pm 6.8 \mathrm{~s}(n=11)$, which differed significantly $(p<0.001$, Bonferroni/Dunn test) from that measured for control mice $(162.2 \pm 13.0 \mathrm{~s}, n=9)$ in the extinction test (Fig. 1C). Moreover, when compared with the control group, the reduction in the duration of the freezing response from extinction training to the extinction test did not change in mice injected with 3 or $10 \mathrm{mg} / \mathrm{kg}$ PEPA, but the reduction was highly significant in mice injected with $30 \mathrm{mg} / \mathrm{kg}$ PEPA (Fig. $1 D)(n=11 ; p=0.005$, Bonferroni/Dunn test). These results suggest that injection of $30 \mathrm{mg} / \mathrm{kg}$ PEPA $15 \mathrm{~min}$ before the extinction training facilitates the formation and subsequent development of extinction learning. This effect of PEPA depended on the extinction training because the effect of PEPA was not detected in the extinction test when the extinction training was omitted (Fig. $1 E, F)[p=0.969$; vehicle (V) vs PEPA, $n=10]$. This result suggests that PEPA during the extinction training is required for its action.

\section{PEPA does not inhibit the initial retrieval of fear memory during extinction training}

It has been reported that extinction learning is triggered by retrieval of fear memory (Ouyang and Thomas, 2005). Thus, we tested whether PEPA suppresses retrieval of fear memory. For this purpose, we analyzed the time course of freezing response when mice were exposed to the operation box during extinction training. PEPA was injected 15 min before extinction training, and the duration of freezing response was measured every $60 \mathrm{~s}$ during extinction training. The duration did not differ significantly for the initial two bins ( $p=0.746$ and 0.074 for bins 1 and 2 , respectively) (Fig. $1 G$ ) between control and $30 \mathrm{mg} / \mathrm{kg}$ PEPAinjected mice. However, the duration was markedly shorter for PEPA-injected mice after the third bin (bins 3-5) (Fig. 1G), and the values for bins $3-5$ were significantly smaller for the PEPAinjected mice ( $p=0.002,0.002$, and 0.001 , respectively, twotailed Student's $t$ test). In contrast, the pattern of the time course of freezing response was completely different in the case of the extinction test (Fig. $1 \mathrm{H}$ ). The duration of freezing response for the PEPA-injected mice was significantly shorter at each time point compared with control mice ( $p<0.02$ for all points) (Fig. $1 H)$. These results suggest that PEPA does not inhibit initial retrieval of fear memory.

\section{The effect of PEPA on extinction learning is mediated by AMPA receptors}

We tested whether the effect of PEPA on extinction learning is mediated by AMPA receptors. For this purpose, mice were injected with NBQX (1 mg/kg, s.c.), an antagonist of AMPA recep- 
A Conditioning $\stackrel{24 \mathrm{~h}}{\longrightarrow}$ Extinction training $\stackrel{48 \mathrm{~h}}{\longrightarrow}$ Extinction test

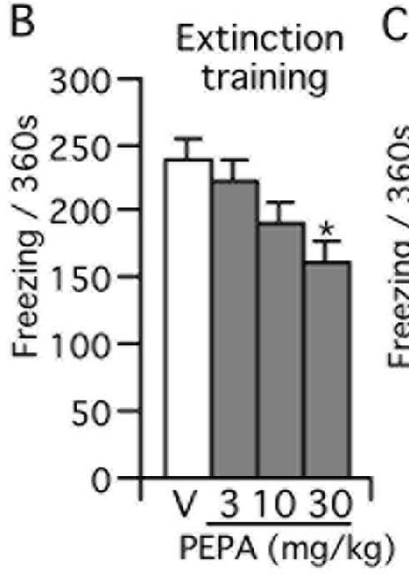

E
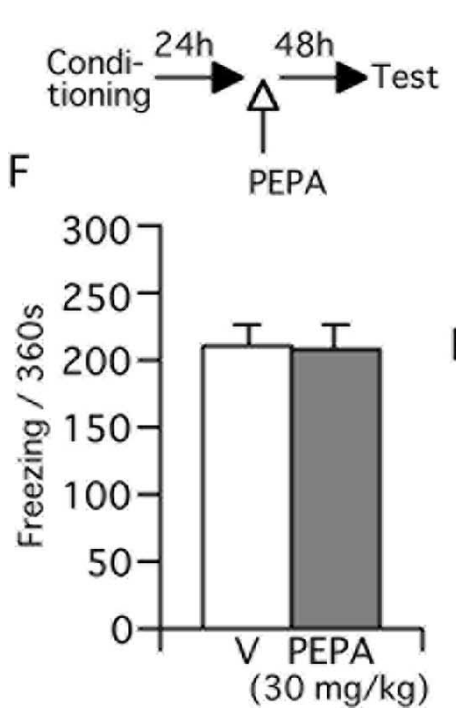

C

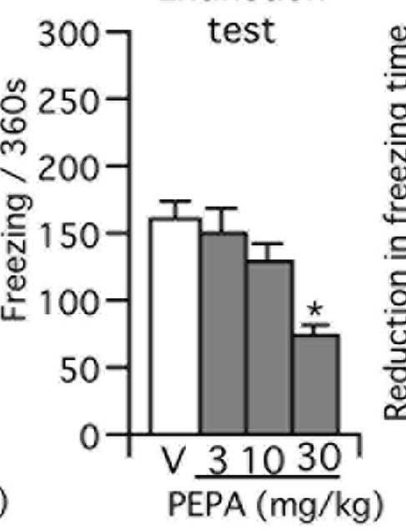

G

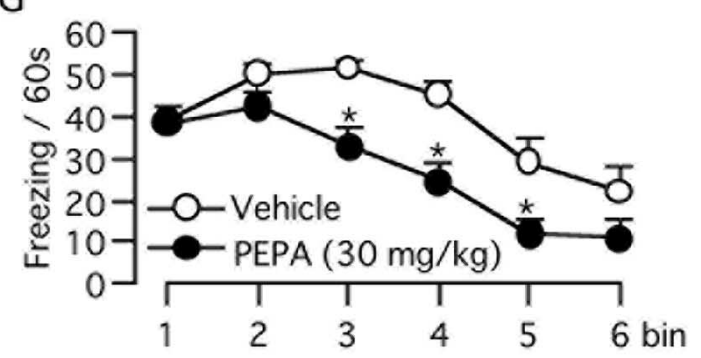

$\mathrm{H}$

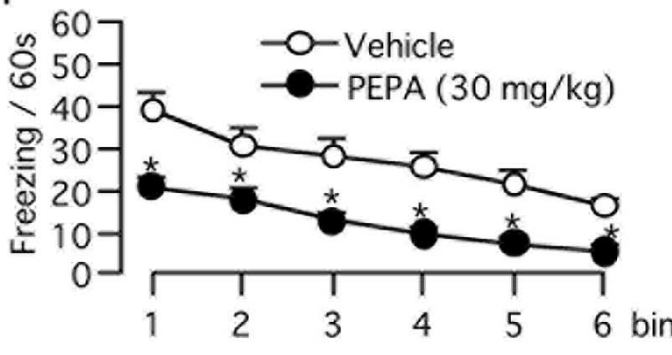

Figure 1. PEPA facilitates extinction learning. $\boldsymbol{A}$, Experimental design for $\boldsymbol{B}-\boldsymbol{D}$. Mice were trained for the contextual fear conditioning and tested $24 \mathrm{~h}$ (extinction training) and $72 \mathrm{~h}$ (extinction test) later. PEPA or vehicle (V) was injected (i.p.) 15 min before extinction training. $\boldsymbol{B}, \boldsymbol{C}$, Dose-dependent reduction of freezing time during extinction training $(\boldsymbol{B})$ and extinction test (C). Data represent mean + SEM ( $n=9-11$ for each group). $\boldsymbol{D}$, Reduction in freezing time between the extinction training and extinction test based on the data in $\boldsymbol{B}$ and $\boldsymbol{C}$. $\boldsymbol{E}$, Experimental design for $\boldsymbol{F}$. Mice were trained for the contextual fear conditioning and tested $72 \mathrm{~h}$ later (test) without extinction training. PEPA or vehicle were injected $24 \mathrm{~h}$ after conditioning. $\boldsymbol{F}$, PEPA did not reduce freezing time in the test when extinction training was skipped $\left(n=10\right.$ each). ${ }^{*}$ Statistically significant $(p=0.002$ vs $V$ in $\boldsymbol{B} ; p<0.001$ vs V in $\boldsymbol{C}$, and $p=0.005$ vs V in $\boldsymbol{D}$, Bonferroni/Dunn test). $\boldsymbol{G}, \boldsymbol{H}$, Changes in freezing time during the 360 s extinction training $(\boldsymbol{G})$ and test $(\boldsymbol{H})$ in groups injected with PEPA $(30 \mathrm{mg} / \mathrm{kg}, n=11)$ or vehicle $(n=9)$. Data used in $\boldsymbol{B}$ and $\boldsymbol{C}$ were reanalyzed for the time course of changes in freezing time (bin $=60 \mathrm{~s}$ ). *Statistically significant ( $p=0.002,0.002$, and 0.001 for bins 3-5, respectively, vs $V$ in $\boldsymbol{G}$, and $p<0.02$ for all vs $V$ in $\boldsymbol{H}$, two-tailed Student's $t$ test).

tors, 60 min before PEPA injection, and the extinction training and test were performed (Fig. 2A). We similarly injected saline (subcutaneously) as the control for NBQX treatment. The timing of injection and concentration of NBQX were determined by referring to a previous report (Lu and Wehner, 1997). The saline/ PEPA-injected mice displayed a significantly shorter freezing time than saline/vehicle-injected mice in both the extinction training and test $(p<0.001$ and $p=0.002$ for training and test, respectively, Bonferroni/Dunn test) (Fig. $2 B)$. These results were similar to those in Figure 1. The NBQX/PEPA-injected mice, however, displayed a significantly longer freezing response than saline/PEPAinjected mice $(p=0.004$ and 0.001 for training and test, respectively, Bonferroni/ Dunn test), and the values were comparable with those of control mice. The freezing response of NBQX/vehicle-injected mice did not differ significantly from saline/vehicle-injected mice $(p=0.614$ and 0.784 for training and test, respectively, Bonferroni/Dunn test). These results suggest that pretreatment with NBQX antagonizes the action of PEPA on extinction learning.

PEPA does not influence locomotion and anxiolytic drug-sensitive behavior Because augmented locomotion may be one of the indirect factors that attenuate freezing behavior, we examined whether PEPA stimulates locomotion. Mouse locomotion was measured in an open-field arena for $5 \mathrm{~min}$, and $30 \mathrm{mg} / \mathrm{kg}$ PEPA was injected $15 \mathrm{~min}$ before the test. The locomotion, as assessed by the total distance the mouse traveled for $5 \mathrm{~min}$, did not differ between control and PEPA-injected groups (Fig. $3 A$ ). In addition, because any compound that changes the anxiety state of mice may be difficult to assess with respect to its true effect on the inhibitory learning process (Bouton et al., 1990) (but see Castellano and McGaugh 1990), we tested the effect of PEPA on mouse behaviors, namely thigmotaxis and head dipping, that are sensitive to anxiolytic drugs such as benzodiazepines (Takeda et al., 1998; Kong et al., 2006). Thigmotaxis is a characteristic of walking close to the walls when a mouse is placed in an open-field arena (for review, see Prut and Belzung, 2003). Head-dipping is an exploratory behavior in which a mouse inserts its head into a hole prepared in an open-filed arena (Takeda et al., 1998). We compared these behaviors between mice injected with PEPA (30 mg/kg) or vehicle and found that the percentage of the time that mice spent at the perimeter in an open-field test (Fig. $3 B$ ) and the number of head-dipping behaviors in a hole-board test (Fig. 3C) were almost identical between PEPA- and vehicle-injected mice. These results suggest that PEPA does not induce detectable changes in locomotion or in the anxiety state of mice.

Neural circuit activation by PEPA is more potent in the mPFC than in the BLA and hippocampal CA1 field

We attempted to specify the brain region that is important for the effect of PEPA on extinction learning. As mentioned earlier, the 
A

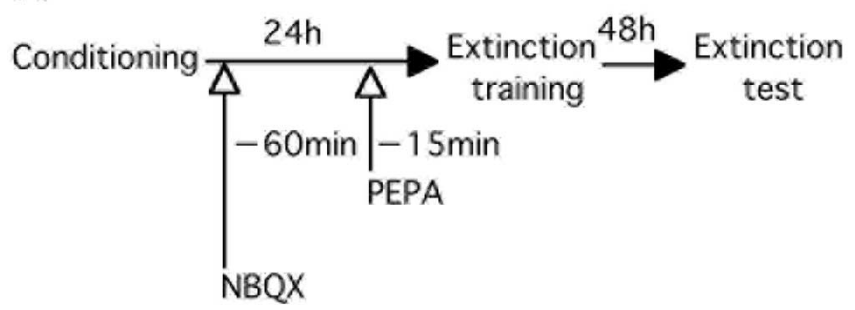

B

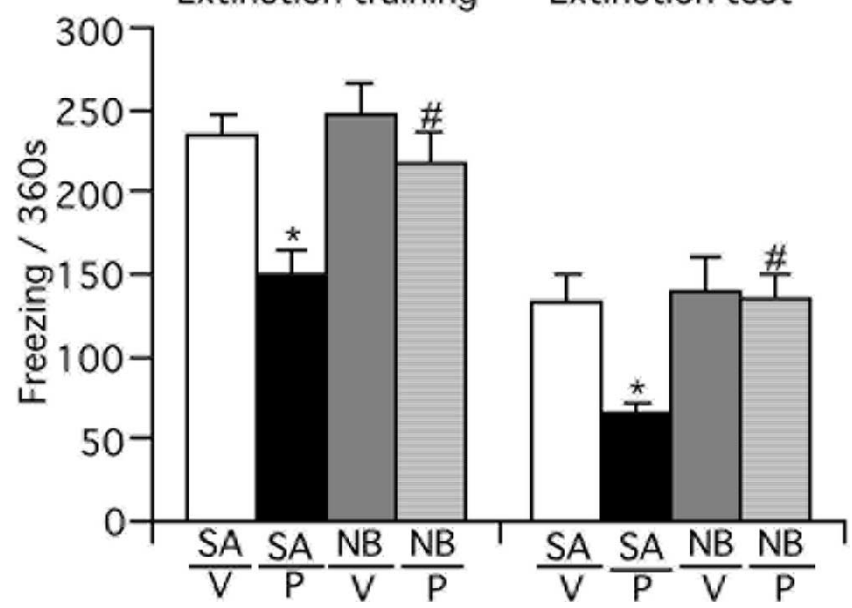

Figure 2. The effect of PEPA on extinction learning is mediated by AMPA receptors. $A$, Experimental design. Mice were injected with saline (SA) or NBQX (NB, $1 \mathrm{mg} / \mathrm{kg}$, s.c.) $60 \mathrm{~min}$ before vehicle (V) or PEPA (P; $30 \mathrm{mg} / \mathrm{kg}$ ) injection (i.p.) and extinction training and testing were performed. $B, N B Q X$ antagonizes the effect of PEPA on extinction learning. *Statistically significant ( $p<0.001$ and $p=0.002$ vs SA/V in extinction training and test, respectively, Bonferroni/Dunn test; ${ }^{\#} p=0.004$ and 0.001 vs SA/P in extinction training and test, respectively, Bonferroni/Dunn test). Error bars indicate + SEM.
A

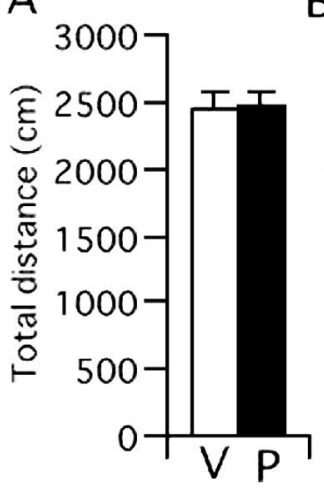

B

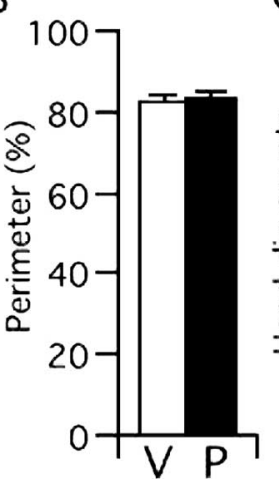

C

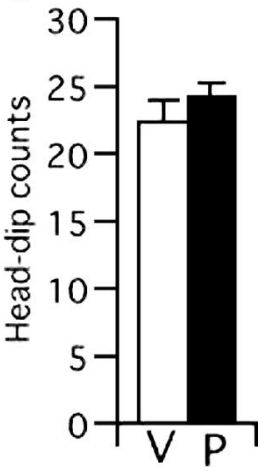

Figure 3. PEPA does not influence locomotion and anxiolytic drug-sensitive behavior. Mice were injected (i.p.) with vehicle (V) or PEPA (P;30 mg/kg) 15 min before the open-field test and hole-board test. $A, B, C$, Locomotion assessed by total distance that mice traveled for $5 \mathrm{~min}(\boldsymbol{A})$, the percentage of time that the mice spent in the perimeter of the open-field $(\boldsymbol{B})$, and the number of head-dipping behaviors in the hole-board test $(\boldsymbol{C})$. None of the values differed significantly between the groups injected with vehicle ( $n=6$ for all tests) or PEPA ( $n=7$ for all tests). Error bars indicate + SEM.

neural circuit consisting of the BLA, the hippocampus CA1/subiculum, and the mPFC is thought to be the main structure important for extinction learning of contextual fear memory. Thus, we tested the effect of PEPA on the synaptic response recorded from neurons in brain slices prepared from mouse mPFC, BLA, and hippocampal CA1 field.
Figure $4 \mathrm{~A}$ shows synaptic currents recorded from layer $\mathrm{V}$ pyramidal cells in response to electrical stimulation of the $\mathrm{mPFC}$ layer II in mouse brain slices. Two types of synaptic current modulation were recorded when PEPA $(50 \mu \mathrm{M})$ was applied to the slices. One was a simple augmentation of the synaptic currents, which we called type I modulation (Fig. $4 A$, mPFC type I). The AUB ratio of this type of modulation by PEPA was 1.25-2.26, and this type of modulation was observed in 11 of 17 cells recorded $(65 \%)$. The other type involved potent epileptiform activity with long latency, which we called type II modulation (Fig. 4 A, mPFC type II). Type II modulation was observed in 5 of 17 cells tested (29\%). The epileptiform activity was not generated by the perfusion of PEPA alone without electrical stimulation of layer II, suggesting that this activity is elicited by synaptic activation. Application of AMPA receptor antagonist, CNQX $(20 \mu \mathrm{M})$, completely abolished the epileptiform activity, as well as the initial small synaptic response, in cells showing type II modulation (Fig. 4A, mPFC type II), suggesting that AMPA receptors participated in the generation of the epileptiform activity. We defined the cases in which the AUB ratio was $<1.1$ as "no modulation" (NM), in which no obvious change in the current responses was evident. In the mPFC, 1 of 17 cells recorded was found to have NM. Augmentation of the synaptic currents by PEPA was dose dependent (Fig. $4 B$ ) in cells showing type I modulation, and $50 \mu \mathrm{M}$ PEPA yielded a mean AUB ratio of $1.56 \pm 0.11(n=11)$.

Figure $4 C$ shows synaptic currents recorded from BLA pyramidal cells in response to electrical stimulation of the external capsule in mouse brain slices. When PEPA $(50 \mu \mathrm{M})$ was applied to the slices, 11 of the 13 cases tested $(85 \%)$ were found to have NM (AUB ratio, 0.91-1.09) (Fig. 4C, BLA NM). The remaining two cells $(15 \%)$ exhibited the simple augmentation of the synaptic response (AUB ratio, 1.58 and 1.81), which is similar to type I modulation in the mPFC (Fig. $4 C$, BLA type I). In no case did PEPA cause the epileptiform activity.

Figure $4 D$ shows synaptic currents recorded from neurons in the CA1 pyramidal layer in response to electrical stimulation of the stratum radiatum in mouse hippocampal slices. When PEPA $(50 \mu \mathrm{M})$ was applied to the slices, 9 of the 12 cases tested $(75 \%)$ were found to have NM (AUB ratio, 0.88-1.09) (Fig. 4D, CA1 $\mathrm{NM})$. The remaining three cells (25\%) exhibited the simple augmentation of the synaptic response (AUB ratio, 1.44, 2.11, and 2.92), which is similar to type I modulation in the mPFC (Fig. $4 D$, CA1-type I). In no case did PEPA cause the epileptiform activity. We applied cyclothiazide (CYZ), a flip splice variant-preferring potentiator of AMPA receptors (Partin et al., 1994), to the three cells that were found to have NM. Typical results are illustrated in Figure $4 D$ (NM-CYZ); the application of CYZ very potently enhanced synaptic currents in these cells.

Figure $4 E$ summarizes the effect of PEPA on synaptic currents in the three brain regions. PEPA showed much higher potency on synaptic currents in the mPFC than in the BLA and CA1. In particular, PEPA characteristically elicited epileptiform activity only in the mPFC. These electrophysiological data suggest that neural circuit activation by PEPA is more potent in the mPFC than in the BLA and hippocampal CA1 field.

\section{Quantitative PCR analysis of AMPA receptor mRNA expression}

As mentioned above, PEPA preferentially acts on flop variants and GluR3/4 subunits (Sekiguchi et al., 1997, 2002). In contrast, CYZ preferentially acts on flip variants (Partin et al., 1994). The potent augmentation by CYZ of synaptic currents that are insensitive to PEPA prompted us to hypothesize that AMPA receptors 
A
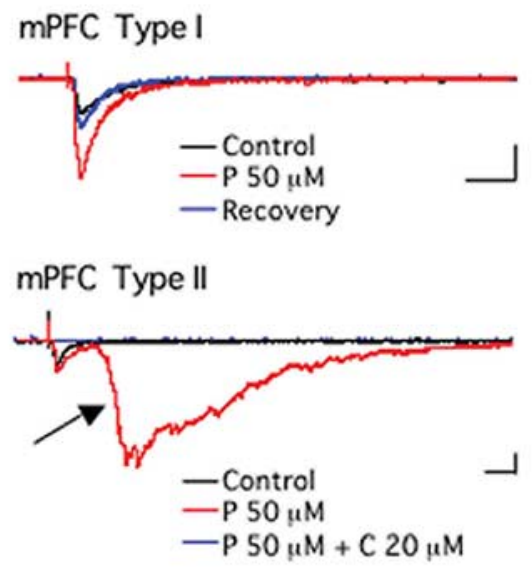

B

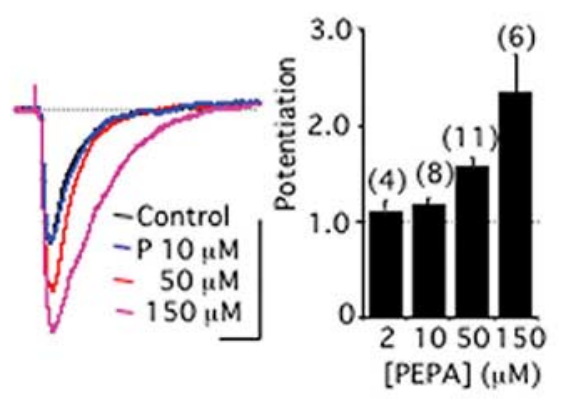

C

\section{BLANM}

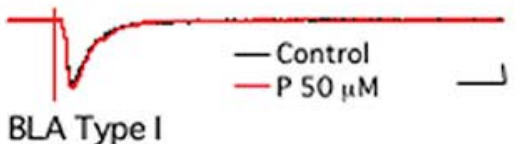

D

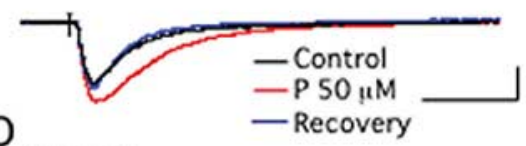

CA1 NM
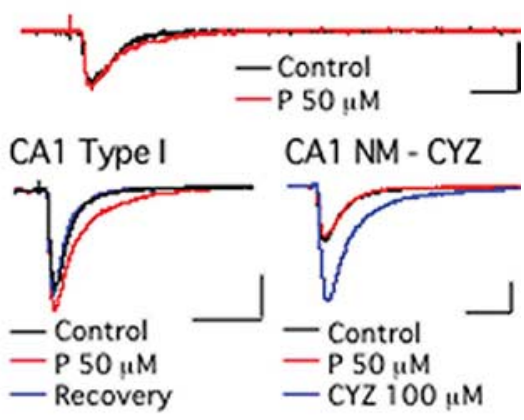

E

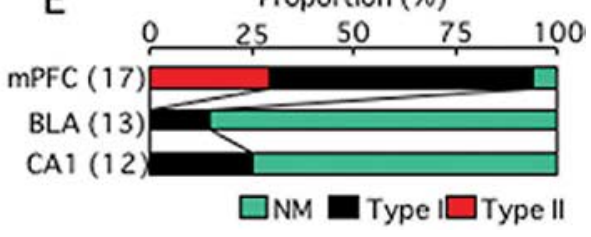

Figure 4. PEPA more potently activates the neural circuit in the $\mathrm{MPFC}$ than in the BLA or hippocampal CA1 field. $A$, mPFC-type I, An example of type I modulation by PEPA of synaptic currents recorded from layer V pyramidal cells in response to electrical stimulation of the mPFC layer II in mouse brain slices. A, mPFC-type II, An example of type II modulation by PEPA of similar synaptic currents. The arrow indicates epileptiform activity. C indicates CNQX. B, Dose-dependent augmentation of synaptic currents by PEPA in cells showing type I modulation. C, BLA-NM, An example of synaptic currents that were not affected by PEPA. NM, no modulation. The synaptic currents were recorded from a BLA pyramidal cell in response to electrical stimulation of the external capsule in mouse brain slices. BLA-type I, An example of type I modulation by PEPA of similar synaptic currents. D, CA1-NM, An example of synaptic currents that were not affected by PEPA. The synaptic currents were recorded from a neuron in the CA1 pyramidal layer in response to electrical stimulation of stratum radiatum in mouse hippocampal slices. CA1-type I, An example of type I modulation by PEPA of similar synaptic currents. NM-CYZ, An example of similar synaptic currents that were not affected by PEPA but potently enhanced by CYZ. Calibrations: $20 \mathrm{~ms}, 100 \mathrm{pA}$. $E$, Summary of the action of PEPA $(50 \mu \mathrm{m})$ in the mPFC, BLA, and CA1-field (the total number of cells tested is shown in parentheses). The proportion of the cells exhibiting NM, type I, and type II modulations is indicated by color in each bar. Error bars indicate + SEM.

in the MPFC have subunit and splice variant compositions that are particularly sensitive to PEPA, which would explain why PEPA preferentially activates neural circuits in the mPFC. Indeed, it is reported that GluR3-flop, one of PEPA-preferring subunits, is relatively abundantly expressed in the rat PFC when compared with GluR1-flip, GluR1-flop, and GluR3-flip (Stine et al., 2001). To test this idea, we performed quantitative PCR for RNA samples prepared from mouse $\mathrm{MPFC}$, the amygdala (including the BLA), and the hippocampus.

Figure $5 A$ shows normalized expression levels of each AMPA receptor subunit in the three brain regions tested. The expression level of GluR3 was significantly higher in the mPFC than in the amygdala and hippocampus ( $p=0.017$ vs the amygdala, and $p<$ 0.001 vs the hippocampus, two-tailed Student's $t$ test). The expression level of GluR4 was significantly higher in the mPFC than in the hippocampus ( $p<0.001$, two-tailed Student's $t$ test). Because PEPA prefers GluR3/4, it is possible that the higher electrophysiological activity of PEPA in the MPFC compared with the hippocampus is attributable to the higher relative expression level of GluR3/4 in the mPFC. In the case of the amygdala, however, the expression level of GluR4 was not significantly different from the MPFC and the difference in the expression level of GluR3 was not so remarkable as the case of the hippocampus. Thus, we compared the relative expression of flop variants between the $\mathrm{mPFC}$ and amygdala. As Figure $5 B$ shows, the percentage of the flop variant in all subunits was higher in the mPFC than in the amygdala $(p<0.001$ for GluR1, $p=$ 0.006 for GluR2, $p=0.009$ for GluR3, and $p=0.012$ for GluR4, two-tailed Student's $t$ test; data for four mice). Because PEPA preferentially acts on flop variants compared with flip variants, it is possible that more abundant expression of flop variants in the mPFC than in the amygdala contributes to the higher activity of PEPA in the mPFC than the BLA.

\section{Intra-mPFC injection of PEPA} facilitates extinction learning

The electrophysiological results obtained above suggested the strong participation of the mPFC in facilitating the action of PEPA on extinction learning. To confirm this, we microinjected PEPA into the $\mathrm{mPFC}$ of fear-conditioned mice and performed extinction training and testing (Fig. 6A). For comparison, we also conducted bilateral microinjection of PEPA into the amygdala (Fig. 6A) using a similar number of mice and the same concentration of PEPA. We could not observe any abnormal behaviors such as seizures in either of the mice injected with PEPA into the mPFC or amygdala. The location of the tip of injection cannulas in the mPFC is indicated in Figure $6 B$, and $C$ shows the duration of freezing response of mice microinjected with vehicle or PEPA $(0.02 \mu \mathrm{g})$ into the mPFC. The PEPA-injected mice ( $n=5)$ showed significantly shorter freezing time than the vehicle-injected mice $(n=6)$ both in the extinction training and test $(p=0.004$ and 0.029 , respectively, two-tailed Student's $t$ test). In both the extinction training and test, the reduction of freezing time by PEPA was similar between intraperitoneal injection of $30 \mathrm{mg} / \mathrm{kg}$ PEPA (Fig. $1 \mathrm{~B}, C$ ) and intra-mPFC injection (32.4\% for i.p. and $32.3 \%$ for intra-PFC in extinction training; $54.4 \%$ for i.p. and $60.7 \%$ for intra-mPFC in the extinction test). Figure $6, D$ and $E$, shows the time course of freezing response of mice received intra-mPFC injection in the extinction training and test, respectively. The patterns of the time course were almost similar to those obtained in intraperitoneal injection (Fig. 1G,H). In contrast, the intra-amygdala injection of PEPA (Fig. $6 \mathrm{~F}$ for the cannula location) did not significantly change the freezing time in either extinction training or extinction test when compared with vehicle-injected mice (Fig. $6 G$ ) $[p=0.120$ and 0.250, vehicle-injected group $(n=5)$ vs PEPA-injected group $(n=6)$ in extinction training and extinction test, respectively, two-tailed Student's $t$ test]. However, although not significant, mice injected 
with PEPA into the amygdala showed shorter freezing time in mean both in extinction training and the extinction test (Fig. 6G). Therefore, these results suggest that PEPA induces more potent facilitation of extinction with injection into the $\mathrm{mPFC}$ than injection into the amygdala.

\section{Discussion}

The main finding of our present study is that extinction learning for contextual fear memory is facilitated by PEPA, a potentiator of AMPA receptors (Fig. 1). This facilitation occurs through AMPA receptors (Fig. 2) without preventing initial fear memory retrieval (Fig. 1). PEPA does not affect locomotion or anxiolytic drugsensitive behavior of mice (Fig. 3). We also found that PEPA somewhat selectively and potently activates circuit activity in the mPFC (Fig. 4), and that an intra-mPFC injection of PEPA facilitates extinction much more potently than an intraamygdala injection (Fig. 6), suggesting that the $\mathrm{MPFC}$ is a major site in which PEPA acts to facilitate fear extinction.

The amygdala and hippocampus play a central role in forming contextual fear memory (for review, see LeDoux, 2000; Maren, 2001). The information flow from the hippocampus CA1 field or subiculum to the BLA followed by that from the BLA to central amygdala $(\mathrm{CeA})$ is thought to be necessary for contextual fear memory formation (Maren and Fanselow, 1995; for review, see LeDoux, 2000; Maren, 2001). The projection from CeA to the periaqueductal gray matter (PAG) is critical for the freezing response (LeDoux et al., 1988). In contrast, the excitatory projections from the $\mathrm{mPFC}$ to the amygdala play a critical role in extinction learning (Morgan et al., 1993; Morgan and LeDoux, 1995; Garcia et al., 1999; Quirk et al., 2000, 2003; Rosenkranz and Grace, 2001). There are two models on the mechanism underlying extinction by the projection (for review, see SotresBayon et al., 2004). One is a model that the excitatory projection elicits feedforward inhibition for BLA principal neurons via BLA inhibitory interneurons (Rosenkranz and Grace, 2001), and another is a model that the projection elicits feedforward inhibition for CeA projection neurons via inhibitory neurons in the intercalated cell masses (Quirk et al., 2003). These inhibitions attenuate activation of CeA projection neurons, which suppress the CeA-PAG pathways to decrease the freezing response (for review, see Sotres-Bayon et al., 2004). Our present finding that a drug that potently activates the neuronal circuits in the MPFC facilitates extinction learning is in agreement with this significant role of the $\mathrm{MPFC}$ in extinction learning. In particular, it is characteristic that PEPA selectively elicits epileptiform activity in the mPFC. The generation of this type of activity increases spike firings, and the robust increment in spike firings in the infralimbic region of the $\mathrm{mPFC}$ reportedly occurs in response to reexposure of rats to the CS (Milad and Quirk, 2002). Therefore, it is possible that PEPA facilitates extinction learning by enhancing the generation of epileptiform activity in the mPFC. This specu- lation is favored by previous reports which suggest no effect of intra-amygdala infusion of AMPA receptor antagonists on extinction (Falls et al., 1992; Lin et al., 2003). Extinction learning is triggered by retrieval of fear memory in response to the context presentation (Ouyang and Thomas, 2005), and the hippocampus CA1 field may be critical for contextual fear memory retrieval (Hall et al., 2001). Our result that PEPA does not prevent initial fear memory retrieval (Fig. $1 G$ ) can be explained by the low abundance of PEPA-sensitive neurons in the CA1 pyramidal cell layer.

Two factors can be pointed out for the relatively selective activation of the mPFC by PEPA. One is the relatively abundant expression of PEPA-preferring AMPA receptor subunits and splice variants in the mPFC, as shown by quantitative PCR (Fig. 5 ). The subunit and splice variant composition of AMPA receptors depends on the expression level of the subunits and variants in a cell. The expression of the GluR3/4 subunits, which are preferred by PEPA, is higher in the MPFC than in the hippocampus, and the expression of flop variants, which are preferred by PEPA, is higher in the mPFC than in the amygdala and, thus, it is reasonable to assume that PEPA-preferring AMPA receptors are much more abundant in the MPFC than in the CA1-field and amygdala. The potentiation of AMPA receptors by PEPA would be more pronounced under such conditions. Another factor underlying the relatively selective activation of the MPFC by PEPA is the specificity of neural circuits in the MPFC. It has been reported 

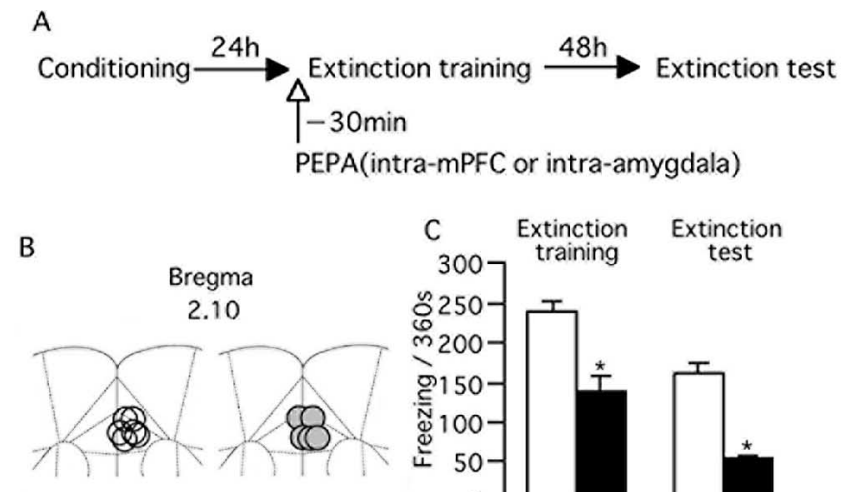

D
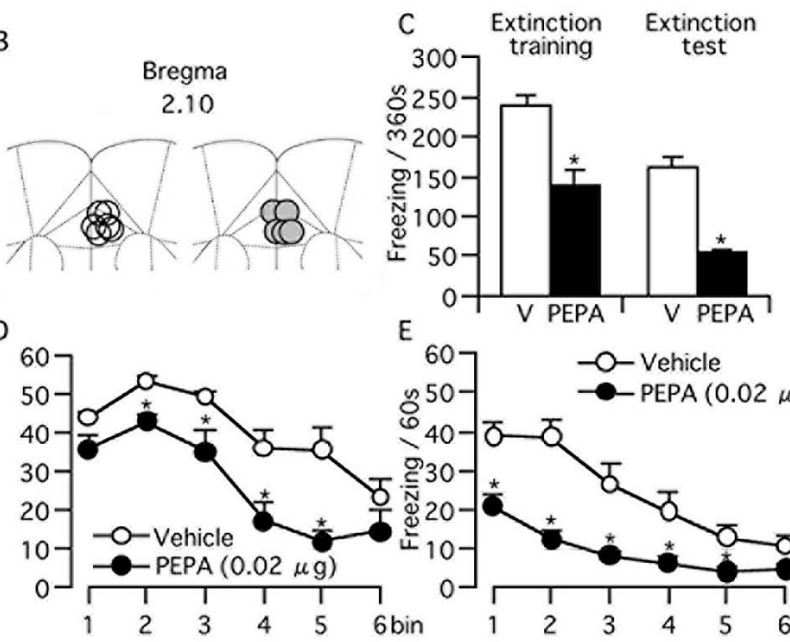

E
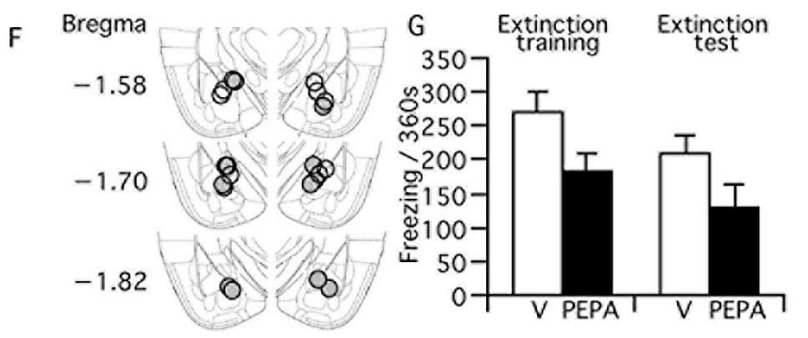

Figure 6. Intra-mPFC injection of PEPA $(0.02 \mu \mathrm{g}) 30$ min before extinction training facilitates extinction learning. $\boldsymbol{A}$, Experimental design. Mice were trained for the contextual fear conditioning and tested $24 \mathrm{~h}$ (extinction training) and $72 \mathrm{~h}$ (extinction test) later. PEPA or vehicle $(V)$ were microinjected into the $\mathrm{mPFC}$ or amygdala 30 min before extinction training. $\boldsymbol{B}$, Location of the injection cannulas within the MPFC. Open circles, Vehicle; shaded circles, PEPA. C, Freezing time of mice microinjected with vehicle $(n=6)$ or PEPA $(n=5)$ into the mPFC in extinction training and test. *Statistically significant ( $p=0.004$ and 0.029 vs V in extinction training and test, respectively, two-tailed Student's t test). $\boldsymbol{D}, \boldsymbol{E}$, Changes in freezing time during the 360 s extinction training $(\boldsymbol{D})$ and test $(\boldsymbol{E})$ in groups injected with vehicle or PEPA. Data used in C were reanalyzed for the time course of changes in freezing time (bin $=60 \mathrm{~s})$. *Statistically significant ( $p=0.004,0.017,0.027$, and 0.014 for bins $2-5$, respectively, vs V in $\boldsymbol{D}$, and $p<$ 0.04 for all vs $V$ in $\boldsymbol{H}$, two-tailed Student's $t$ test). $\boldsymbol{F}$, Location of the injection cannulas within the amygdala. Open circles, vehicle; shaded circles, PEPA. G, Freezing time of mice microinjected with vehicle $(n=6)$ or PEPA $(n=5)$ into the amygdala in extinction training and test. The values in vehicle- and PEPA-injected groups are not significantly different in either extinction training or extinction test ( $p=0.120$ and 0.250 in extinction training and test, respectively, two-tailed Student's $t$ test). Error bars indicate + SEM.

that layer $\mathrm{V}$ pyramidal neurons in the $\mathrm{mPFC}$ form a specific microcircuit (Wang et al., 2006). Specifically, 12\% of the layer V pyramidal neurons form recurrent connections with other layer V pyramidal neurons, and $47 \%$ of the recurrent connections are reciprocal. In the hippocampus CA1 field, only $1 \%$ of the pyramidal cells form recurrent connections with each other (Deuchars and Thomson, 1996). The abundant recurrent connections among layer $\mathrm{V}$ pyramidal neurons can thus elicit population firings of connected pyramidal neurons in response to excitatory inputs. Under this condition, the PEPA-mediated enhancement of excitatory synaptic responses in layer $\mathrm{V}$ pyramidal neurons would increase the proportion of neurons that generate action potentials, which facilitates population firings through the activation of recurrent connections. The characteristic feature of
PEPA action in the mPFC, namely the generation of epileptiform activity, can be explained by the presence of this amplification cascade based on the circuit characteristics of the mPFC.

In the present study, subcutaneous injection of NBQX alone did not affect extinction (Fig. 2). This, however, does not necessarily mean that intact (basic) activity of AMPA receptors in the $\mathrm{mPFC}$ is not important for extinction. The timing and dose of NBQX administration has not been examined in detail in the present study. Moreover, a higher dose of NBQX such as 30 $\mathrm{mg} / \mathrm{kg}$ is reported to affect locomotion in rats (Danysz et al., 1994). Therefore, it seems that more detailed analysis including the intra-mPFC infusion of AMPA receptor antagonists is needed to establish the role of intact activity of AMPA receptors in extinction.

It has been reported that the function of the medial and orbital PFCs is attenuated during PTSD symptom provocation (Bremner, 1999) and that the function of the anterior cingulate cortex, a subregion of $\mathrm{mPFC}$, in PTSD patients is reduced during extinction training for conditioned stimuli (Bremner et al., 2005). The reduction in the volume of the $\mathrm{mPFC}$ and attenuation in the reactivity of the $\mathrm{mPFC}$ in $\mathrm{PTSD}$ patients also has been reported (Shin et al., 2006). Given that the mPFC plays a crucial role in extinction learning, as mentioned above, a compound such as PEPA that facilitates extinction learning through the activation of the $\mathrm{mPFC}$ constitutes a potential drug candidate for CBT for complex anxiety disorders such as PTSD. To date, D-cycloserine (an NMDA receptor agonist/potentiator) (Walker et al., 2002), yohimbine (an $\alpha 2$-adrenoceptor antagonist) (Cain et al., 2006), AM404 (an inhibitor of endogenous cannabinoid breakdown and reuptake) (Chhatwal et al., 2005), and sulpiride (a D2 dopamine receptor antagonist) (Ponnusamy et al., 2005) have been reported to facilitate extinction learning for fear memory. These drugs are thought to facilitate the inhibitory association between the US and CS within the amygdala, but there is no drug that is specified to act on the mPFC. In fact, intra-amygdala injection of $\mathrm{D}$-cycloserine is sufficient to facilitate extinction learning. Therefore, our results, especially those obtained from the intra-mPFC and intra-amygdala microinjections of PEPA, suggest the first example of a compound that facilitates extinction learning by mainly acting on the mPFC. Thus, PEPA (or its derivatives or similar compounds) may provide the basis for the discovery of new drugs that facilitate extinction learning and CBT for psychiatric disorders such as PTSD.

\section{References}

Amat J, Baratta MV, Paul E, Bland ST, Watkins LR, Maier SF (2005) Medial prefrontal cortex determines how stressor controllability affects behavior and dorsal raphe nucleus. Nat Neurosci 8:365-371.

Berman DE, Dudai Y (2001) Memory extinction, learning anew, and learning the new: dissociations in the molecular machinery of learning in cortex. Science 291:2417-2419.

Blanchard RJ, Blanchard DC (1972) Innate and conditioned reactions to threat in rats with amygdaloid lesions. J Comp Physiol Psychol $81: 281-290$.

Bouton ME (2002) Context, ambiguity, and unlearning: sources of relapse after behavioral extinction. Biol Psychiatry 52:976-986.

Bouton ME, Kenney FA, Rosengard C (1990) State-dependent fear extinction with two benzodiazepine tranquilizers Behav Neurosci 104:44-55.

Bouton ME, Rosengard C, Achenbach GG, Peck CA, Brooks DC (1993) Effects of contextual conditioning and unconditional stimulus presentation on performance in appetitive conditioning. Q J Exp Psychol B 46:63-95.

Bremner JD (1999) Alterations in brain structure and function associated with post-traumatic stress disorder. Semin Clin Neuropsychiatry 4:249-255.

Bremner JD, Vermetten E, Schmahl C, Vaccarino V, Vythilingam M, Afzal N, Grillon C, Charney DS (2005) Positron emission tomographic imaging of neural correlates of a fear acquisition and extinction paradigm in 
women with childhood sexual-abuse-related post-traumatic stress disorder. Psychol Med 35:791-806.

Cain CK, Blouin AM, Barad M (2006) Adrenergic transmission facilitates extinction of conditional fear in mice. Learn Mem 11:179-187.

Castellano C, McGaugh JL (1990) Effects post-training bicuculline and muscimol on retention: lack of state dependency. Behav Neural Biol 54:156-164.

Chhatwal JP, Myers KM, Ressler KJ, Davis M (2005) Regulation of gephyrin and $\mathrm{GABA}_{\mathrm{A}}$ receptor binding within the amygdala after fear acquisition and extinction. J Neurosci 22:7892-7902.

Danysz W, Essmann U, Bresink I, Wilke R (1994) Glutamate antagonists have different effects on spontaneous locomotor activity in rats. Pharmacol Biochem Behav 48:111-118.

Davis M, Whalen PJ (2001) The amygdala: vigilance and emotion. Mol Psychiatry 6:13-34

Davis M, Myers KM, Chhatwal J, Ressler KJ (2006) Pharmacological treatments that facilitate extinction of fear: relevance to psychotherapy. NeuroRx 3:82-96.

Deuchars J, Thomson AM (1996) CA1 pyramid-pyramid connections in rat hippocampus in vitro: dual intracellular recordings with biocytin filling. Neuroscience 74:1009-1018.

Falls WA, Miserendino MJ, Davis M (1992) Extinction of fear-potentiated startle: blockade by infusion of an NMDA antagonist into the amygdala. J Neurosci 3:854-863.

Fanselow MS (1998) Pavlovian conditioning, negative feedback, and blocking: mechanisms that regulate association formation. Neuron 20:625-627.

Garcia R, Vouimba RM, Baudry M, Thompson RF (1999) The amygdala modulates prefrontal cortex activity relative to conditioned fear. Nature 402:294-296.

Hall J, Thomas KL, Everitt BJ (2001) Cellular imaging of zif268 expression in the hippocampus and amygdala during contextual and cued fear memory retrieval: selective activation of hippocampal CA1 neurons during the recall of contextual memories. J Neurosci 21:2186-2193.

Hestrin S, Nicoll RA, Perkel DJ, Sah P (1990) Analysis of excitatory synaptic action in pyramidal cells using whole-cell recording from rat hippocampal slices. J Physiol (Lond) 422:203-225.

Kong WX, Chen SW, Li YL, Zhang YJ, Wang R, Min L, Mi X (2006) Effects of taurine on rat behaviors in three anxiety models. Pharmacol Biochem Behav 83:271-276.

LeDoux JE (2000) Emotion circuits in the brain. Annu Rev Neurosci 23:155-184.

LeDoux JE, Iwata J, Cicchetti P, Reis DJ (1988) Different projections of the central amygdaloid nucleus mediate autonomic and behavioral correlates of conditioned fear. J Neurosci 10:1062-1069.

Lin CH, Yeh SH, Lu HY, Gean PW (2003) The similarities and diversities of signal pathways leading to consolidation of conditioning and consolidation of extinction of fear memory. J Neurosci 23:8310-8317.

Lu Y, Wehner JM (1997) Enhancement of contextual fear-conditioning by putative ( \pm )- $\alpha$-3-hydroxy-5-methylisoxazole-4-propionic acid (AMPA) receptor modulators and $N$-methyl-D-aspartate (NMDA) receptor antagonists in DBA/2J mice. Brain Res 768:197-207.

Maren S (2001) Neurobiology of Pavlovian fear conditioning. Annu Rev Neurosci 24:897-931.

Maren S, Fanselow MS (1995) Synaptic plasticity in the basolateral amygdala induced by hippocampal formation stimulation in vivo. J Neurosci 15:7548-7564.

Maren S, Quirk GJ (2004) Neuronal signaling of fear memory. Nat Rev Neurosci 5:844-852.

Mayer ML, Westbrook GL, Guthrie PB (1984) Voltage-dependent block by $\mathrm{Mg}^{2+}$ of NMDA responses in spinal cord neurones. Nature 309:261-263.

Milad MR, Quirk GJ (2002) Neurons in medial prefrontal cortex signal memory for fear extinction. Nature 420:70-74.

Morgan MA, LeDoux JE (1995) Differential contribution of dorsal and ventral medial prefrontal cortex to the acquisition and extinction of conditioned fear in rats. Behav Neurosci 109:681-688.

Morgan MA, Romanski LM, LeDoux JE (1993) Extinction of emotional learning: contribution of medial prefrontal cortex. Neurosci Lett 163:109-113.

Myers KM, Davis M (2002) Behavioral and neural analysis of extinction. Neuron 36:567-584.
Nakagawa T, Iino M, Sekiguchi M, Wada K, Ozawa S (1999) Potentiating effects of 4-[2-(phenylsulfonylamino)ethylthio]-2,6-difluoro-phenoxyacetamide (PEPA) on excitatory synaptic transmission in dentate granule cells. Neurosci Res 35:217-223.

O’Neill MJ, Bleakman D, Zimmerman DM, Nisenbaum ES (2004) AMPA receptor potentiators for the treatment of CNS disorders. Curr Drug Targets CNS Neurol Disord 3:181-189.

Ouyang M, Thomas SA (2005) A requirement for memory retrieval during and after long-term extinction learning. Proc Natl Acad Sci USA 102:9347-9352

Ozawa S, Iino M, Abe M (1991) Excitatory synapse in the rat hippocampus in tissue culture and effects of aniracetam. Neurosci Res 16:6634-6647.

Partin KM, Patneau DK, Mayer ML (1994) Cyclothiazide differentially modulates desensitization of AMPA receptor splice variants. Mol Pharmacol 46:129-138.

Paxinos G, Franklin KB (2001) The mouse brain in stereotaxic coordinate, Ed 2. San Diego: Academic.

Ponnusamy R, Nissim HA, Babara M (2005) Systemic blockade of D2-like dopamine receptors facilitates extinction of conditioned fear in mice. Learn Mem 12:399-406.

Prut L, Belzung C (2003) The open field as a paradigm to measure the effects of drugs on anxiety-like behaviors: a review. Eur J Pharmacol 463:3-33.

Quirk GJ, Russo GK, Barron JL, Lebron K (2000) The role of ventromedial prefrontal cortex in the recovery of extinguished fear. J Neurosci 20:6225-6231.

Quirk GJ, Likhtik E, Pelletier JG, Pare D (2003) Stimulation of medial prefrontal cortex decreases the responsiveness of central amygdala output neurons. J Neurosci 23:8800-8807.

Rosenkranz JA, Grace AA (2001) Dopamine attenuates prefrontal cortical suppression of sensory inputs to the basolateral amygdala of rats. J Neurosci 21:4090-4103.

Sekiguchi M, Fleck MW, Mayer ML, Takeo J, Chiba Y, Yamashita S, Wada K (1997) A novel allosteric potentiator of AMPA receptors: 4-2(phenylsulfonyl-amino)ethylthio-2,6-difluorophenoxyacetamide. J Neurosci 15:5760-5771.

Sekiguchi M, Takeo J, Harada T, Morimoto T, Kudo Y, Yamashita S, Kohsaka S, Wada K (1998) Pharmacological detection of AMPA receptor heterogeneity by use of two allosteric potentiators in rat hippocampal cultures. Br J Pharmacol 123:1294-1303.

Sekiguchi M, Nishikawa K, Aoki S, Wada K (2002) A desensitizationselective potentiator of AMPA-type glutamate receptors. Br J Pharmacol 136:1033-1041.

Shin LM, Rauch SL, Pitman RK (2006) Amygdala, medial prefrontal cortex, and hippocampal function in PTSD. Ann NY Acad Sci 1071:67-79.

Sommer B, Keinanen K, Verdoorn TA, Wisden W, Burnashev N, Herb A, Kohler M, Takagi T, Sakmann B, Seeburg PH (1990) Flip and flop: a cell-specific functional switch in glutamate-operated channels of the CNS. Science 249:1580-1585.

Sotres-Bayon F, Bush DEA, LeDoux JE (2004) Emotional perseveration: an update on prefrontal-amygdala interactions in fear extinction. Learn Mem 11:525-535.

Stine CD, Lu W, Wolf ME (2001) Expression of AMPA receptor flip and flop mRNAs in the nucleus accumbens and prefrontal cortex after neonatal ventral hippocampal lesions. Neuropsychopharmacology 24:253-266.

Takamatsu I, Sekiguchi M, Wada K, Sato T, Ozaki M (2005) Propofolmediated impairment of CA1 long-term potentiation in mouse hippocampal slices. Neurosci Lett 389:129-132.

Takeda H, Tsuji M, Matsumiya T (1998) Changes in head-dipping behavior in the hole-board test reflect the anxiogenic and/or anxyolytic state in mice. Eur J Pharmacol 350:21-29.

Takeda H, Tsuji M, Miyamoto J, Matsuya J, Iimori M, Matsumiya T (2003) Caffeic acid produces antidepressive- and/or anxiolytic-like effects through indirect modulation of the alpha $1 \mathrm{~A}$-adrenoceptor system in mice. NeuroReport 14:1067-1070.

Walker DL, Ressler KJ, Lu KT, Davis M (2002) Facilitation of conditioned fear extinction by systemic infusions of D-cycloserine as assessed with fear-potentiated startle in rats. J Neurosci 22:2343-2351.

Wang Y, Markram H, Goodman PH, Berger TK, Ma J, Goldman-Rakic PS (2006) Heterogeneity in the pyramidal network of the medial prefrontal cortex. Nat Neurosci 9:534-542. 